\title{
Washington State Department of Transportation Energy Efficiency Guidelines For Small Buildings
}
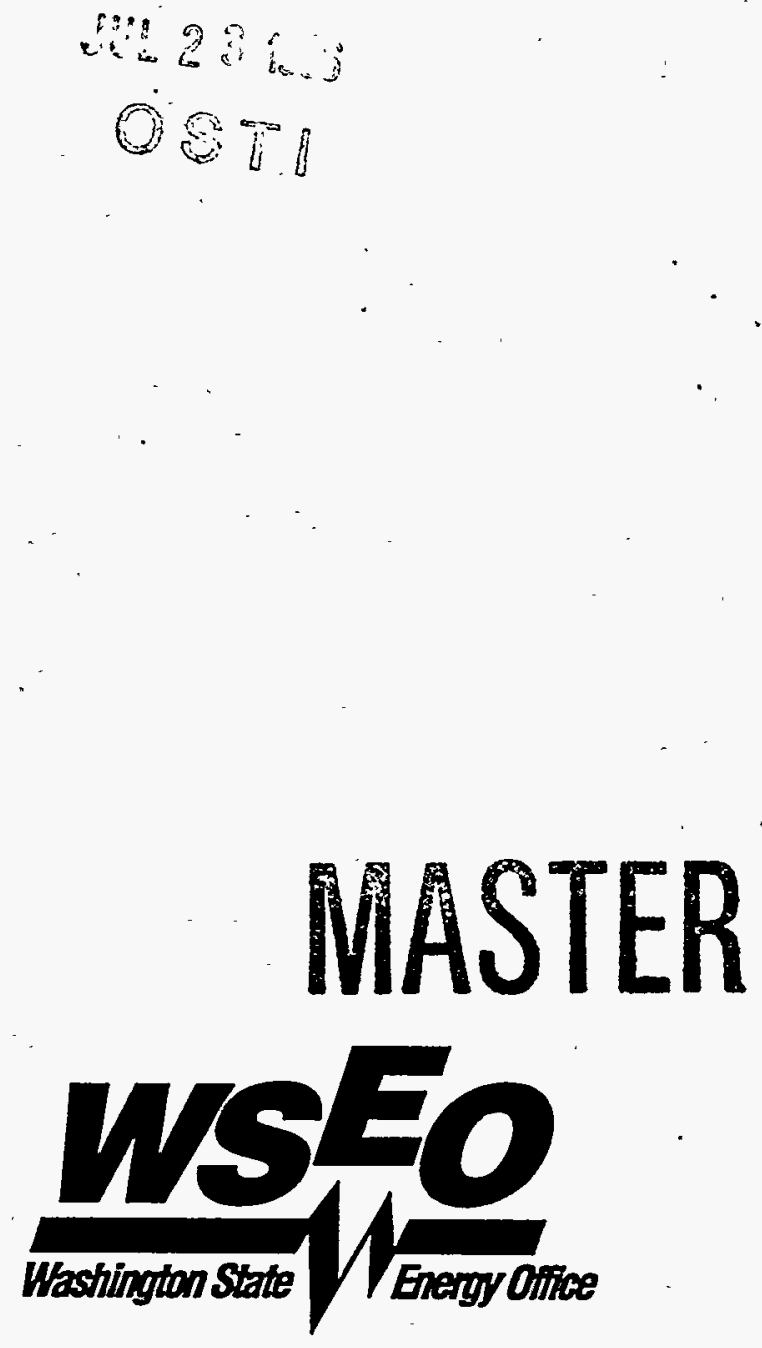



\section{Washington State Department of Transportation Energy Efficiency Guidelines For Small Buildings}

March 1995

WSEO \#95-081

This report can be made available in in another format for people with disabilities. Please call (360) 956-2068. TDD users call (360) 956-2218.

Note: The telephone area code will change January 15, 1995. After this date, use area code 360 .

Printed on recycled paper
Prepared by:

Washington State Energy Office 925 Plum St. SE, Building \#4

Olympia, WA 98504-3165

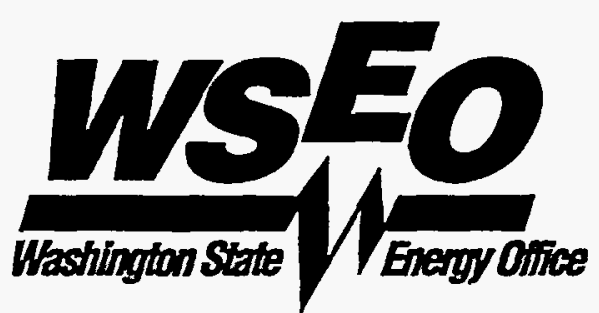


This document wi11 be made available through the National Technical Information Service. 


\section{DISCLAIMER}

Portions of this document may be illegible in electronic image products. Images are produced from the best available original document. 


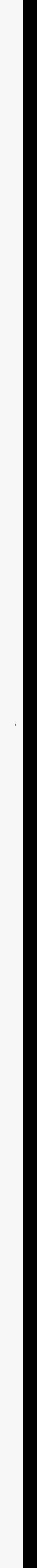




\section{DISCLAIMER}

Portions of this document may be illegible in electronic image products. Images are produced from the best available original document. 



\section{Acknowledgments}

The Washington State Energy Office acknowledges the foresight of the Washington State Department of Transportation's facility management team for proposing and funding this guidebook. 



\section{Preface}

Washington State law (RCW 39.35) requires that a life-cycle-cost analysis be performed in the early design stages of major state building projects. We believe that smaller projects deserve equal attention because they represent a significant proportion of overall operating costs. Prior to these guidelines, however, no document addressed this group of buildings. In addition, despite recent improvements, building energy codes still amount to minimum requirements.

Government should seek to build longer-term energy and economic goals, as well as be a good steward of our natural resources and an example for the rest of society. In this spirit, WSEO produced the following guidelines under contract \#92-35 with the Washington State Department of Transportation. 



\section{Contents}

Acknowledgments

Preface vi

$\begin{array}{ll}\text { 1. Introduction } & \mathbf{1 - 1}\end{array}$

2. Energy Guidelines Methodology $2-1$

3. Building Envelope Considerations 3-1

A. Prescriptive Options 3-1

B. Special Considerations for Office Spaces 3-2

C. Special Considerations for Shops \& Heated Storage 3-3

D. Special Considerations for "Unheated" Storage Spaces 3-3

4. Heating, Ventilating and Air Conditioning 4-1

A. Heating and Air Conditioning Equipment Performance Standards 4-1

B. Outdoor Air Ventilation Requirements 4-5

C. Heat Recovery $4-6$

D. Special HVAC Considerations for Offices . $4-6$

E. Special HVAC Considerations for Shops 4-6

F. Fuel Selection 4-7

G. Other Considerations in Selecting HVAC Systems * ${ }^{*}$

1. Equipment Sizing 4-9

2. Staging Equipment 4-9

3. Air Conditioning Equipment 4-9

4. Infrared Heaters 4-9

5. Other Radiant Heaters $\quad 4-10$

6. Hydronic Heat Pumps 4-10

H. Suggested HVAC Configurations 4-10

5. Lighting 5-1

A. Lighting Densities and Light Levels 5-1

B. Specific Lighting Recommendations for Offices a 5-1

C. Specific Recommendations for Shop Lighting 5-1

D. Specific Recommendation for Exterior Lighting 5-2

6. Building Commissioning 6-1

7. General Issues $\quad$ 7-1

A. Resource Management

B. Co-Locating

C. Equipment Replacement

8. Summary 



\section{Introduction}

This document provides energy efficiency guidelines for the construction and remodel of small buildings owned by the Washington State Department of Transportation (DOT). For the purpose of these guidelines "small buildings" are defined as those under 25,000 square feet. However, many of the guidelines can also be used for larger buildings.

DOT is responsible for 641 buildings totaling 2.2 million square feet and consuming approximately $\$ 1,087,500$ dollars in energy costs each year. Building types covered by these guidelines are small offices, shop buildings, and heated and unheated storage.

These building types can be expected to vary greatly in both the distribution and magnitude of energy use. Figure 1 demonstrates the typical energy use for each of these types of buildings.

Figure 1

Energy Use for Different Building Types

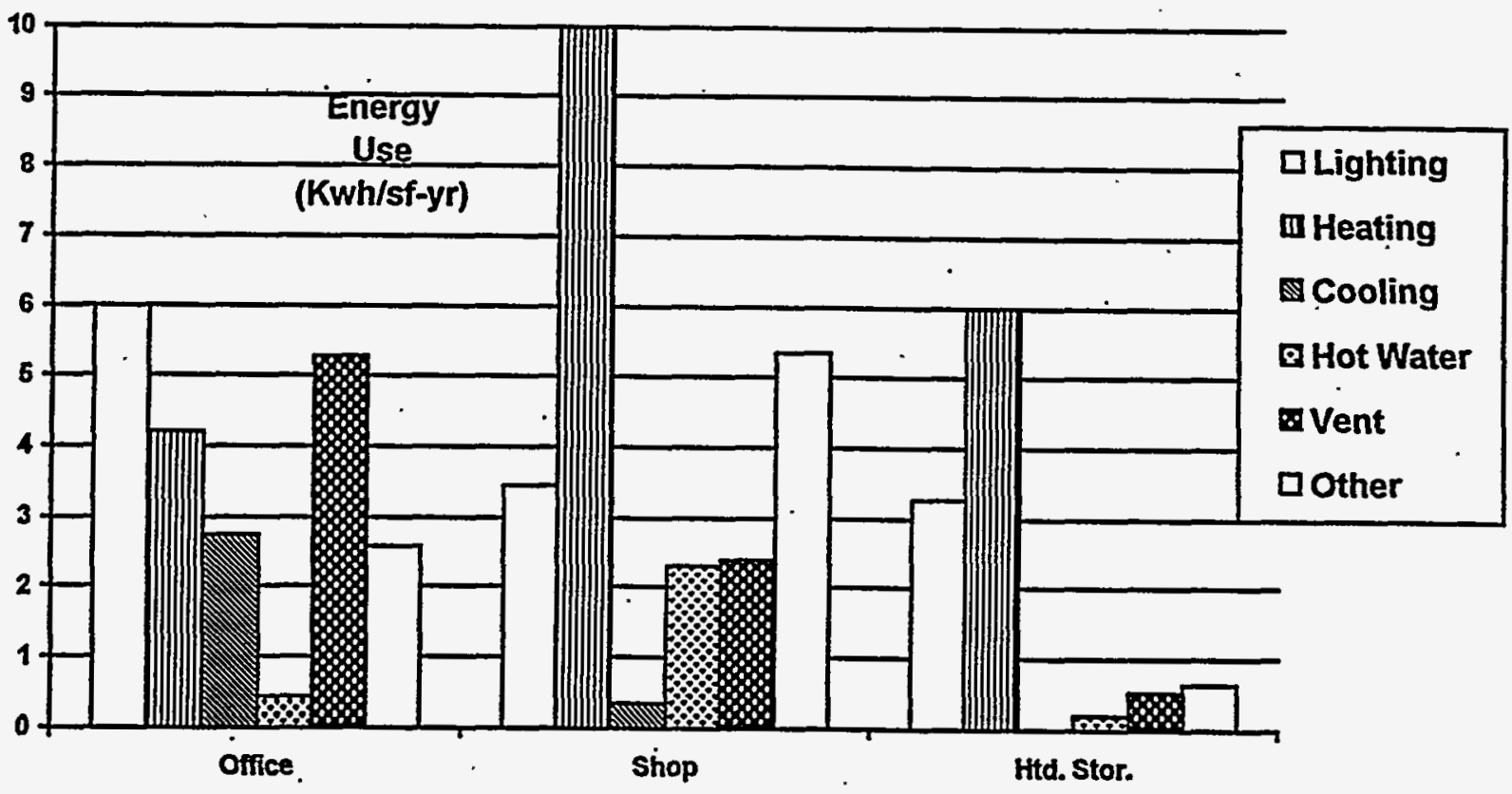





\section{Energy Guidelines Methodology}

These energy guidelines are based on the Washington State Energy Code (WSEC) which became effective April 1, 1994. However, these guidelines slightly exceed the minimum WSEC requirements for envelope and mechanical systems. The WSEC requirements for lighting, heat recovery and ventilation are adequate and will be followed without improvement.

The Washington State Energy Office recommends higher standards for the envelope and mechanical systems because of specific characteristics of many DOT buildings (e.g., remote locations, expense and difficulty of maintenance, locations in areas with higherthan-average energy costs). These guidelines will, in most cases, improve the life-cycle cost of DOT buildings.

If a 25-year life cycle cost study shows that efficiency improvements are truly cost effective, the state will consider allocating the additional expense for the efficiency improvements. These guidelines should be considered a starting point. We encourage designers to verify cost effectiveness and customize particular items if appropriate for the project under consideration. It also should be noted that comfortable working conditions directly affect worker productivity, and therefore comfort should never be compromised. This would include adequate lighting, temperature and humidity control, sound, and air quality. 



\section{Building Envelope Considerations}

\section{A. Prescriptive Options}

A number of paths are open for WSEC envelope compliance. The paths include prescriptive, component performance, and analysis. A fourth method, an alternative component performance path, uses the ASHRAE 90.1 compliance tool, a computer program known as ENVSTD (Envelope Standard). This fourth compliance path, allowed for non-electric resistance ${ }^{1}$ heated buildings only, tends to qualify buildings with low glazing percentages that do not meet the regular component performance criteria. For simplicity, the following guidelines focus on the prescriptive requirements' path.

Table 1, DOT Envelope Prescriptive Options, modifies the WSEC tables 13-1 and 13-2 which give prescriptive minimum insulation values for all commercial building types. WSEO makes the following changes to the WSEC tables based on the types of buildings DOT ordinarily constructs:

$\checkmark \quad$ Table 1 requires more insulation than code in areas in which WSEO considers it cost effective for DOT.

$\checkmark \quad$ The table sets the maximum glazing percentage (window and skylight area divided by the gross wall area) at $15 \%$ of gross wall. This can be exceeded and still meet code, but doing so will generally require a more energy efficient and higher priced glazing product.

$\checkmark \quad$ Straight resistance heating systems are not included as a mechanical prescriptive; therefore that option is omitted from the envelope table. Using resistance for spot heating or heat pump back up is acceptable.

\footnotetext{
' Electric resistance heated buildings are defined as those in which electric resistance heat capacity exceeds one watt per square foot of gross floor area. The exceptions are electric resistance elements in heat pump systems and those in VAV terminal (reheat) boxes.
} 
Table 1

DOT Envelope Prescriptive Options

\begin{tabular}{|c|c|c|c|c|}
\hline & Option 1 & Option 2 & Option 3 & Option 4 \\
\hline $\begin{array}{l}\text { Roof } \\
\text { Zone } 1\end{array}$ & $\begin{array}{l}\text { R-30 Batt } \\
\text { R-21 Rigid }\end{array}$ & $\begin{array}{l}\text { R-38 Batt } \\
\text { R-30 Rigid }\end{array}$ & $\begin{array}{l}\text { R-38 Batt } \\
\text { R-30 Rigid }\end{array}$ & $\begin{array}{l}\text { R-38 Batt } \\
\text { R-30 Rigid }\end{array}$ \\
\hline $\begin{array}{l}\text { Roof } \\
\text { Zone } 2\end{array}$ & $\begin{array}{l}\text { R-38 Batt } \\
\text { R-25 Rigid }\end{array}$ & $\begin{array}{l}\text { R-38 Batt } \\
\text { R-30 Rigid }\end{array}$ & $\begin{array}{l}\text { R-38 Batt } \\
\text { R-30 Rigid }\end{array}$ & $\begin{array}{l}\text { R-38 Batt } \\
\text { R-30 Rigid }\end{array}$ \\
\hline Wall & R-19 Wood Frame. & $\begin{array}{c}\text { R-19 Steel frame } \\
\text { or panel }\end{array}$ & $\begin{array}{l}\text { Concrete block with } \\
\text { R-10 Insulation in or } \\
\text { out }\end{array}$ & $\begin{array}{l}* * \mathrm{CMU} \text { with } \\
\text { internal insulation } \\
\text { tested to } \mathrm{U}=0.11 \\
\text { (entire wall) }\end{array}$ \\
\hline Windows* & $\mathrm{U}=0.60$ & $\mathrm{U}=0.60$ & $\mathrm{U}=0.60$ & $\mathrm{U}=0.60$ \\
\hline Skylight* & $\mathrm{U}=1.20$ & $\mathrm{U}=1.20$ & $\mathrm{U}=1.20$ & $\mathrm{U}=1.20$ \\
\hline $\begin{array}{l}\text { Max. Glazing \% } \\
\text { of wall area }\end{array}$ & $15 \%$ & $15 \%$ & $15 \%$ & $15 \%$ \\
\hline Opaque Door & $\mathrm{U}=0.50$ & $\mathrm{U}=0.50$ & $\mathrm{U}=0.50$ & $\mathrm{U}=0.50$ \\
\hline $\begin{array}{l}\text { Floor } \\
\text { Zone } 1\end{array}$ & R-19 & $\mathrm{R}-19$ & $\mathrm{R}-19$ & $\mathrm{R}-19$ \\
\hline $\begin{array}{l}\text { Floor } \\
\text { Zone 2* }\end{array}$ & $\mathrm{R}-21$ & $\mathrm{R}-21$ & $\mathrm{R}-21$ & $\mathrm{R}-21$ \\
\hline Slab & $\mathrm{R}-10$ & $\mathrm{R}-10$ & R-10 & $\mathrm{R}-10$ \\
\hline \multicolumn{5}{|c|}{$\begin{array}{l}\text { *These values assume the heating type is other than electric resistance. If not the case, these values may } \\
\text { have to be improved per tables } 13-1 \text { and 13-2 of the WSEC. } \\
\text { **ASTM C-236-87 } \\
\text { Note: Zone } 1 \text { and Zone } 2 \text { are defined in the WSEC }\end{array}$} \\
\hline
\end{tabular}

\section{B. Special Considerations for Office Spaces}

DOT office spaces are usually dedicated buildings or accessory to buildings whose major function is a shop or storage. From an energy standpoint, offices generally have high internal loads during occupied periods and are often in the cooling mode most of the time. It is a common misconception that less wall and roof insulation will lower the energy bill for cooling-dominated buildings. This argument does not hold up for two reasons:

1. Office buildings in the Northwest spend far more time actively heating than cooling. State offices are occupied approximately $25 \%$ of the time. With airside economizers, they should be in "free cooling" mode for all but 5001000 hours of the year. During unoccupied hours internal loads will be less than envelope losses, and therefore in the heating mode from mid-October through April, approximately 3500 hours.

2. Cooling equipment is sized for a design load, which invariably occurs on a warm afternoon (work) day. A thin building envelope results in a higher. design load, and larger equipment. The larger size equipment results in higher air flows, more on-and-off cycling of equipment, and higher cooling and heating bills. The bottom line is that a more resistive envelope is always of some benefit from an energy standpoint. 


\section{Special Considerations for Shops \& Heated Storage}

Shop buildings occupied by DOT serve an assortment of uses other than just vehicle repair. Most shops are constructed either of concrete masonry or steel construction with a concrete floor. They may have moderately high ceilings and sliding or roll-up type garage doors to access the vehicle service bays. Many ancillary uses of energy occur in the fluid handling systems such as compressed air, lubricants, dust collection, and vehicle exhaust venting systems. Buildings used for heated storage should be built with similar thermal features as an occupied shop building. Ventilation for warehouses is expressed in the code as a CFM/SF. This is taken to mean only during periods of occupied use.

Infiltration through shop doors can be excessive and lead to uncomfortable working conditions and additional energy usage. These doors should be tight fitting when closed. Insulated doors should be used in every new facility. Door replacement should be considered in older facilities. HVAC interlocks should be installed on the doors to prevent heating equipment operation when the door is open. Caution needs to be taken in hydronic systems to prevent accidental freezing.

Separate véhicular storage used for de-icing should be physically separated from the shop with an R-11 wall. This space should always have a separate thermostat.

\section{Special Considerations for "Unheated" Storage Spaces}

The definition of unheated space has changed over the years. In the previous version of the WSEC, buildings less than 3.4 BtuH/sf in heater capacity were considered unheated, and exempt. Under the new WSEC, section 1310.2 states that all spaces shall be considered heated (actually "conditioned") unless they meet the definition for semi-heated. Semi-heated spaces are defined as those with 3-8 BtuH/sf heating capacity (5-12 BtuH/sf in zone 2). They are required to have roof insulation. The code implies (but does not actually state) that a building is "unheated" below the $3 \mathrm{BtuH} / \mathrm{sf}$ threshold (5 BTUH/SF in zone 2). However, WSEC section 1301.2 states that special permission is required by the code official for a building to be considered unheated. The code is not considered to require a semi-heated space to have a heater.

If a building has $3 \mathrm{BTUH} / \mathrm{sf}$ (0.879 watt/sf) or lower heater capacity, section 1301.2 allows owners (with approval of the code jurisdiction) to be exempt from the envelope requirements. Due to the discretionary nature of this, its usefulness is uncertain. The next level of insulation is the "semi heated" building. This means a heater can be included, but no larger than 8 BTUH/SF in zone 1 and 12 BTUH/SF in zone 2. A semi-heated building's roof must be insulated (to a degree) but the walls need not be. A word of caution, however...a building designed to be semi heated is not necessarily fully freeze protected. 



\section{Heating, Ventilating and Air Conditioning}

\section{A. Heating and Air Conditioning Equipment Performance Standards}

The 1994 WSEC contains tables 14-1, 14-2, and 14-3 defining minimum efficiencies for heating and cooling equipment. The numbers are similar to those first proposed for use after 1992 in ASHRAE 90.1-1989. The National Energy Act of 1992 adopted the same efficiencies. Thus, these efficiencies now represent the minimums that can be manufactured and distributed in the U.S.

Considerably more efficient equipment is now available. Therefore, target component efficiencies for DOT buildings shall be $110 \%$ of the values in tables 14.1 and 14.2 of the 1994 WSEC and $105 \%$ of the values in table 14.3 of the 1994 WSEC. WSEO has modified the following tables to reflect the more stringent efficiencies: 
Table 2

Modified WSEC TABLE 14-1

\section{Standard Rating Conditions and Minimum Performance for Air Cooled Unitary Air Conditioners, Heat Pumps, Packaged Terminal Air Conditioners, Warm Air Furnaces, Duct Furnaces and Unit Heaters}

\begin{tabular}{|c|c|c|c|c|c|}
\hline \multirow{2}{*}{ Typupipant } & \multirow{2}{*}{ Oategory } & \multirow{2}{*}{$\begin{array}{l}\text { Sub category \& } \\
\text { Rating Conditions }\end{array}$} & \multicolumn{2}{|c|}{ Minimum Rating g } & \multirow{2}{*}{$\begin{array}{l}\text { Standard } \\
4 \\
0\end{array}$} \\
\hline & & & Steady State & $\begin{array}{l}\text { Seasonalor } \\
\text { Part Loád } \\
\text { Pa }\end{array}$ & \\
\hline \multirow{3}{*}{$\begin{array}{l}\text { Air Conditioners } \\
\text { and Heat Pumps } \\
\text { Cooling }\end{array}$} & $\begin{array}{l}\leq 65,000 \text { Btu/h } \\
\text { Cooling Capacity }\end{array}$ & $\begin{array}{l}\text { Split Systems } \\
\text { Single Package }\end{array}$ & $\begin{array}{l}\text { NA } \\
\text { NA }\end{array}$ & $\begin{array}{l}\text { 11.0 SEER } \\
\text { 10.7 SEER } \\
\end{array}$ & \multirow[t]{2}{*}{$\begin{array}{l}\text { ARI 210/240- } \\
1989\end{array}$} \\
\hline & $\begin{array}{l}>65,000 \text { and } \leq 135,000 \\
\text { Btu/h } \\
\text { Cooling Capacity }\end{array}$ & $\begin{array}{l}\text { All Unitary } \\
\text { Standard Ratings: }\end{array}$ & $\begin{array}{l}\text { 8.9 EER } \\
95^{\circ} \mathrm{F} \mathrm{db}\end{array}$ & $\begin{array}{l}9.1 \mathrm{IPLV} \\
80^{\circ} \mathrm{F} \mathrm{db}\end{array}$ & \\
\hline & $\begin{array}{l}>135,000 \text { and } \leq 760,000 \\
\text { Btu/h } 1\end{array}$ & Air Conditioners & 9.4 EER & 8.3 IPLV & \multirow[t]{3}{*}{ ARI 360-1986 } \\
\hline \multirow[t]{2}{*}{ Ratings } & Cooling Capacity & Heat Pumps & 9.4 EER & 8.3 IPLV & \\
\hline & $\begin{array}{l}>760,000 \mathrm{Btu} / \mathrm{h}^{1} \\
\text { Cooling Capacity }\end{array}$ & $\begin{array}{l}\text { Air Conditioners } \\
\text { Heat Pumps }\end{array}$ & $\begin{array}{l}\text { 9.0 EER } \\
\text { 9.6 EER }\end{array}$ & $\begin{array}{l}\text { 8.3 IPLV } \\
\text { 8.3 IPLV }\end{array}$ & \\
\hline $\begin{array}{l}\text { Packaged Term. } \\
\text { Air Conditioners } \\
\text { \& Heat Pumps } \\
\text { Cooling Ratings }\end{array}$ & All Capacities & $\begin{array}{l}\text { Air Conditioners } \\
\text { and Heat Pumps } \\
\text { Standard/Low Temp: }\end{array}$ & $\begin{array}{l}10.0-(0.16 \mathrm{x} \\
\text { Cap/1000) } \\
\text { EER } \\
95^{\circ} \mathrm{F} \\
\end{array}$ & $\begin{array}{l}12.2-(0.20 \mathrm{x} \\
\text { Cap/1000) } 2,3 \\
\text { EER } \\
82^{\circ} \mathrm{F}\end{array}$ & ARI 310-1990 \\
\hline \multirow[b]{2}{*}{ Heat Pump } & $\begin{array}{l}\leq 65,000 \mathrm{Btu} / \mathrm{h} \\
\text { Cooling Capacity }\end{array}$ & $\begin{array}{l}\text { Split Systems } \\
\text { Single Package }\end{array}$ & & $\begin{array}{l}\text { 6.8 HSPF } \\
\text { 6.6 HSPF }\end{array}$ & \multirow[t]{3}{*}{$\begin{array}{l}\text { ARI 210/240- } \\
1989\end{array}$} \\
\hline & $\begin{array}{l}>65,000 \text { and } \leq 135,000 \\
\text { Btu/h }\end{array}$ & All Unitary & 3.3 COP & $2.2 \mathrm{COP}$ & \\
\hline Heating & Cooling Capacity & Standard Ratings: & $47^{\circ} \mathrm{F} \mathrm{db} / 43^{\circ} \mathrm{F} w b$ & $\begin{array}{l}17^{\circ} \mathrm{F} \mathrm{db} / 15^{\circ} \mathrm{F} \\
w b\end{array}$ & \\
\hline Ratings & $\begin{array}{l}>135,000 \mathrm{Btu} / \mathrm{h} \\
\text { Cooling Capacity }\end{array}$ & Standard Ratings: & $\begin{array}{l}3.2 \mathrm{COP} \\
47^{\circ} \mathrm{F}\end{array}$ & $\begin{array}{l}2.2 \mathrm{COP} \\
17^{\circ} \mathrm{F} \\
\end{array}$ & ARI 365-1986 \\
\hline $\begin{array}{l}\text { Packaged Term. } \\
\text { Heat Pumps } \\
\text { Heating Ratings }\end{array}$ & All Capacities & $\begin{array}{l}\text { Heat Pumps } \\
\text { Standard Ratings: }\end{array}$ & \multicolumn{2}{|c|}{$\begin{array}{c}2.9-(0.026 \times \text { Cap } / 1000)^{3} \\
\text { EER } \\
47^{\circ} \mathrm{F} \mathrm{db} / 43^{\circ} \mathrm{F} \text { wb }\end{array}$} & ARI 380-1990 \\
\hline \multirow{3}{*}{$\begin{array}{l}\text { Warm Air } \\
\text { Furnaces \& } \\
\text { Combination } \\
\text { Furnace/A.C. }\end{array}$} & $<225,000 \mathrm{Btu} / \mathrm{h}$ & $\begin{array}{l}\text { Gas and Oil Fired } \\
\text { Seasonal Ratings: }\end{array}$ & $88 \% \mathrm{E}_{\mathrm{f}}^{4}$ & $86 \%$ AFUE $^{5}$ & $\begin{array}{l}\text { DOE 10CFR } \\
\text { Part430 AppN }\end{array}$ \\
\hline & $\geq 225,000 \mathrm{Btu} / \mathrm{h}$ & $\begin{array}{l}\text { Gas, Max Rating } 6 \\
\text { Gas, Min Rating } 6\end{array}$ & \begin{tabular}{|l}
$88 \% \mathrm{E}_{\mathrm{t}}^{4}$ \\
$86 \% \mathrm{E}_{\mathrm{t}}^{4}$ \\
\end{tabular} & $\begin{array}{l}\text { NA } \\
\text { NA }\end{array}$ & \begin{tabular}{|l} 
ANSI Z21.47- \\
1983 \\
\end{tabular} \\
\hline & $\geq 225,000 \mathrm{Btu} / \mathrm{h}$ & $\begin{array}{l}\text { Oil, Max Rating6 } \\
\text { Oil, Min Rating6 }\end{array}$ & $\begin{array}{l}89 \% \mathrm{E}_{\mathrm{t}}^{4} \\
89 \% \mathrm{E}_{\mathrm{t}}^{4}\end{array}$ & $\begin{array}{l}\text { NA } \\
\text { NA }\end{array}$ & UL 727-1986 \\
\hline \multirow{3}{*}{$\begin{array}{l}\text { Warm-Air } \\
\text { Duct Furnaces } \\
\cdot \\
\text { and Unit Heaters }\end{array}$} & All Size Gas Duct Furnaces & $\begin{array}{l}\text { Max Rated Capacity }{ }^{6} \\
\text { Min Rated Capacity } 6\end{array}$ & $\begin{array}{l}86 \% \mathrm{E}_{\mathrm{f}}^{4} \\
83 \% \mathrm{E}_{\mathrm{t}}^{4}\end{array}$ & $\begin{array}{l}\text { NA } \\
\text { NA }\end{array}$ & $\begin{array}{l}\text { ANSI Z83.9- } \\
1986\end{array}$ \\
\hline & All Size Gas Unit Heaters & $\begin{array}{l}\text { Max Rated Capacity } \\
\text { Min Rated Capacity }\end{array}$ & $\begin{array}{l}86 \% \mathrm{E}_{\mathrm{t}}^{4} \\
81 \% \mathrm{E}_{\mathrm{t}}^{4}\end{array}$ & $\begin{array}{l}\text { NA } \\
\text { NA }\end{array}$ & $\begin{array}{l}\text { ANSI Z83.8- } \\
1985\end{array}$ \\
\hline & All Size Oil Unit Heaters & $\begin{array}{l}\text { Max Rated Capacity } 6 \\
\text { Min Rated Capacity } 6\end{array}$ & $\begin{array}{l}89 \% \mathrm{E}_{\mathrm{t}}^{4} \\
89 \% \mathrm{E}_{\mathrm{t}}^{4}\end{array}$ & $\begin{array}{l}\text { NA } \\
\text { NA }\end{array}$ & UL 731-1988 \\
\hline
\end{tabular}

1. For units that have a heating section, deduct 0.2 from all required EER's and IPLV's.

2. For multi-capacity equipment the minimum performance shall apply to each step provided. Multi-capacity refers to manufacturer published rating for more than one capacity mode allowed by the product's controls.

3. Capacity (Cap) means the rated cooling capacity of the product in Btu/h in accordance with the cited ARI standard. If the unit's capacity is less than $7,000 \mathrm{Btu} / \mathrm{h}$, use $7,000 \mathrm{Btu} / \mathrm{h}$ in the calculation. If the unit's capacity is greater than $15,000 \mathrm{Btu} / \mathrm{h}$, use $15,000 \mathrm{Btw} / \mathrm{h}$ in the calculation.

4. These values apply to non-NAECA equipment. See referenced standard for definition of Thermal efficiency (Et), (100\% - flue losses).

5. To be consistent with National Appliance Energy Conservation Act (NAECA) of 1987 (Public Law 100-12). These values apply to furnace and combination units covered by NAECA.

6. Minimum and maximum ratings as provided for and allowed by the unit's controls. 
Table 3

Modified WSEC TABLE 14-2

Standard Rating Conditions and Minimum Performance for Water and Evaporatively Cooled Unitary Air Conditioners, Heat Pumps, Water Source and Ground Source Heat Pumps, Condensing Units, and Water Chilling Packages

\begin{tabular}{|c|c|c|c|c|c|}
\hline 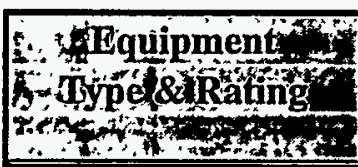 & \multicolumn{2}{|c|}{ 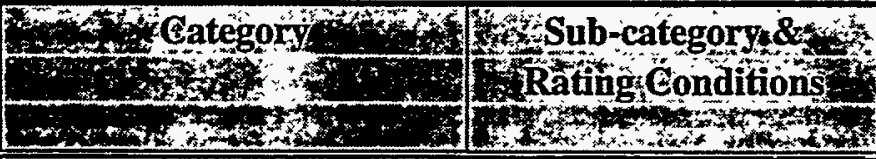 } & \multicolumn{3}{|c|}{ 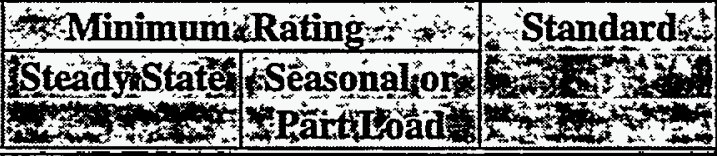 } \\
\hline \multirow{2}{*}{$\begin{array}{l}\text { Evaporatively } \\
\text { Cooled A/Cs \& } \\
\text { Heat Pumps } \\
\text { Cooling Ratings } \\
\end{array}$} & $\begin{array}{l}\leq 65,000 \mathrm{Btu} / \mathrm{h} \\
\text { Cooling Capacity }\end{array}$ & Standard Conditions 1 : & 10.2 EER & 9.4 IPLV & \multirow{2}{*}{$\begin{array}{l}\text { ARI 210/240- } \\
1989 \\
\text { CTI 201-1986 }\end{array}$} \\
\hline & $\begin{array}{l}>65,000 \text { and } \leq 135,000 \mathrm{Btu} / \mathrm{h} \\
\text { Cooling Capacity }\end{array}$ & $\begin{array}{l}\text { Outdoor Conditions: } \\
95^{\circ} \mathrm{F} \mathrm{db} / 75^{\circ} \mathrm{F} \text { wb }\end{array}$ & 11.6 EER & 10.7 IPLV & \\
\hline \multirow{2}{*}{$\begin{array}{l}\text { Water Source } \\
\text { Heat Pump } \\
\text { Cooling Ratings }\end{array}$} & $\begin{array}{l}\leq 65,000 \mathrm{Btu} / \mathrm{h} \\
\text { Cooling Capacity }\end{array}$ & $\begin{array}{l}\text { Standard Conditions } 1: \\
\text { Entering Water }\end{array}$ & \begin{tabular}{|l|}
10.2 EER \\
$85^{\circ} \mathrm{F}$ ewt $^{2}$ \\
\end{tabular} & $\begin{array}{l}11.2 \text { EER } \\
75^{\circ} \mathrm{F} \mathrm{ewt} \\
\end{array}$ & \multirow{2}{*}{$\begin{array}{l}\text { ARI 320-1986 } \\
\text { CTI 201-1986 }\end{array}$} \\
\hline & $\begin{array}{l}>65,000 \text { and } \leq 135,000 \mathrm{Btu} / \mathrm{h} \\
\text { Cooling Capacity }\end{array}$ & $\begin{array}{l}\text { Standard Conditions }{ }^{1} \text { : } \\
\text { Entering Water }\end{array}$ & $\begin{array}{l}11.6 \text { EER } \\
85^{\circ} \mathrm{F} \mathrm{ewt}^{2} \\
\end{array}$ & $\mathrm{NA}$ & \\
\hline $\begin{array}{l}\text { Ground Water } \\
\text { Heat Pump } \\
\text { Cooling Ratings } \\
\end{array}$ & $\begin{array}{l}<135,000 \mathrm{Btu} / \mathrm{h} \\
\text { Cooling Capacity }\end{array}$ & $\begin{array}{l}\text { Standard Conditions } 1: \\
\text { Entering Water }\end{array}$ & $\begin{array}{l}12.1 \text { EER } \\
70^{\circ} \mathrm{F} \text { ewt }\end{array}$ & $\begin{array}{l}\text { 12.7 EER } \\
50^{\circ} \mathrm{F} \mathrm{ewt}^{2}\end{array}$ & ARI 325-1985 \\
\hline \multirow{2}{*}{$\begin{array}{l}\text { Water Cooled } \\
\text { Unitary Air } \\
\text { Conditioners } \\
\text { Cooling Ratings } \\
\end{array}$} & $\begin{array}{l}\leq 65,000 \text { Btu/h } \\
\text { Cooling Capacity }\end{array}$ & $\begin{array}{l}\text { Standard Conditions } 1 \text { : } \\
\text { Entering Water }\end{array}$ & $\begin{array}{l}\text { 10.2 EER } \\
85^{\circ} \mathrm{Fewt}^{2} \\
\end{array}$ & $\begin{array}{l}\text { 9.1 IPLV } \\
75^{\circ} \mathrm{F} \mathrm{ewt} \\
\end{array}$ & \multirow{2}{*}{$\begin{array}{l}\text { ARI 210/240- } \\
1989 \\
\text { CTI 201-1986 }\end{array}$} \\
\hline & $\begin{array}{l}>65,000 \text { and } \leq 135,000 \mathrm{Btu} / \mathrm{h} \\
\text { Cooling Capacity }\end{array}$ & $\begin{array}{l}\text { Standard Conditions } 1 \text { : } \\
\text { Entering Water }\end{array}$ & $\begin{array}{l}11.6 \mathrm{EER} \\
85^{\circ} \mathrm{F} \mathrm{ewt}^{2}\end{array}$ & $\mathrm{NA}$ & \\
\hline $\begin{array}{l}\text { Water/Evap } \\
\text { Cooled Air Cond. } \\
\text { and Heat Pumps } \\
\text { Cooling Ratings }\end{array}$ & $\begin{array}{l}>135,000 \text { Btu/h } \\
\text { Cooling Capacity }\end{array}$ & Standard Conditions ${ }^{1}$ : & $10.6 \mathrm{EER}$ & $9.9 \mathrm{IPLV}$ & $\begin{array}{l}\text { ARI 360-1986 } \\
\text { CTI 201-1986 }\end{array}$ \\
\hline $\begin{array}{l}\text { Air and Water/ } \\
\text { Evap Cooled } \\
\text { Condensing Units } \\
\text { Cooling Ratings } \\
\end{array}$ & $\begin{array}{l}>135,000 \mathrm{Btu} / \mathrm{h} \\
\text { Cooling Capacity }\end{array}$ & $\begin{array}{l}\text { Air Cooled } \\
\text { Water/Evap Cooled }\end{array}$ & $\begin{array}{l}\text { 10.9 EER } \\
\text { 14.2 EER }\end{array}$ & $\begin{array}{l}12.1 \mathrm{IPLV} \\
14.2 \mathrm{IPLV}\end{array}$ & $\begin{array}{c}\text { ARI 365-1987 } \\
\cdot \\
\text { CTI 201-1986 }\end{array}$ \\
\hline $\begin{array}{l}\text { Air and Water } \\
\text { Cooled } \\
\text { Water Chilling }\end{array}$ & $\begin{array}{l}<150 \text { Tons } \\
\geq 150 \text { and }<300 \text { Tons } \\
\geq 300 \text { Tons }\end{array}$ & Water Cooled & $\begin{array}{l}4.2 \mathrm{COP} \\
4.6 \mathrm{COP} \\
5.7 \mathrm{COP}^{4}\end{array}$ & $\begin{array}{l}4.3 \mathrm{IPLV} \\
5.0 \mathrm{IPLV} \\
5.8 \mathrm{IPLV}^{4}\end{array}$ & $\begin{array}{l}\text { ARI 550-90 } \\
\text { ARI 590- } \\
86 \mathrm{pN} \\
\text { CTI 201-1986 }\end{array}$ \\
\hline $\begin{array}{l}\text { Packages } \\
\text { Cooling Ratings }\end{array}$ & $\begin{array}{l}<150 \text { Tons } \\
\geq 150 \text { Tons } \\
\text { All Capacities } \\
\end{array}$ & $\begin{array}{l}\text { Air Cooled } \\
\text { with Condenser } \\
\text { Air Cooled Condenserless }\end{array}$ & \begin{tabular}{|l|}
$3.0 \mathrm{COP}$ \\
$2.8 \mathrm{COP}$ \\
$3.4 \mathrm{COP}$ \\
\end{tabular} & \begin{tabular}{|l|}
$3.1 \mathrm{IPLV}$ \\
$2.8 \mathrm{IPLV}$ \\
$3.5 \mathrm{IPLV}$ \\
\end{tabular} & CTI 201-1986 \\
\hline \multirow{2}{*}{$\begin{array}{l}\text { Water \& Ground- } \\
\text { Water Source } \\
\text { Heat Pumps } \\
\text { Heating Ratings } \\
\end{array}$} & \multirow[t]{2}{*}{$\begin{array}{l}<135,000 \text { Btu/h } \\
\text { Cooling Capacity }\end{array}$} & \begin{tabular}{|l|} 
Water Source \\
Standard Conditions 1 : \\
\end{tabular} & $\begin{array}{l}4.2 \mathrm{COP} \\
70^{\circ} \mathrm{F} \text { ewt } \\
\end{array}$ & $\begin{array}{l}\text { NA } \\
\text { NA } \\
\end{array}$ & ARI 320-1986 \\
\hline & & $\begin{array}{l}\text { Ground Water Source } \\
\text { Standard Conditions } 1 \text { : }\end{array}$ & $\begin{array}{l}3.7 \mathrm{COP} \\
70^{\circ} \mathrm{Fewt}^{2}\end{array}$ & $\begin{array}{l}3.3 \mathrm{COP} \\
50^{\circ} \mathrm{F} \text { ewt }^{2}\end{array}$ & ARI 325-1985 \\
\hline
\end{tabular}

2. ewt: Entering Water Temperature for water cooled heat pumps and air conditioners.

3. Condensing unit requirements are based on single - number rating defined in paragraph 5.1.3.2 of ARI Standard 365.

4. These requirements are reduced to 5.2 COP and 5.3 IPLV, where refrigerants with ozone depletion factors of 0.05 or less are used. No reduction is allowed for standard design systems analyzed under Standard RS-29. 
Table 4

Modified WSEC TABLE 14-3

Standard Rating Conditions and Minimum Performance, Gas- and Oil-Fired Boilers

\begin{tabular}{|c|c|c|c|}
\hline Referenced & $\therefore$ Categorye & Rating Gonditiond & $\begin{array}{l}\text { Minimimim } \\
\text { Performánces }\end{array}$ \\
\hline \multirow{2}{*}{$\begin{array}{c}\text { DOE Test Procedure } \\
10 \text { CFR, Part } 430 \\
\text { AppN }\end{array}$} & $\begin{array}{c}\text { Gas-Fired } \\
<300,000 \text { Btu/h }\end{array}$ & $\begin{array}{c}\text { Seasonal } \\
\text { Rating }\end{array}$ & $\begin{array}{l}\text { AFUE } \\
88 \% 1,3\end{array}$ \\
\hline & $\begin{array}{c}\text { Oil-Fired } \\
<300,000 \mathrm{Btu} / \mathrm{h}\end{array}$ & $\begin{array}{c}\text { Seasonal } \\
\text { Rating }\end{array}$ & $\begin{array}{l}\text { AFUE } \\
88 \% 1\end{array}$ \\
\hline \multirow{2}{*}{$\begin{array}{c}\text { ANSI Z21.13-87 } \\
\text { H.I. Htg. Boiler Std. } 86 \\
\text { ASME PTC4.1-64 } \\
\text { U.L. 795-73 }\end{array}$} & \multirow[t]{2}{*}{$\begin{array}{c}\text { Gas-Fired } \\
\geq 300,000 \mathrm{Btu} / \mathrm{h}\end{array}$} & $\begin{array}{l}\text { 1. Max. Rated Capacity }{ }^{2} \\
\text { Steady-State }\end{array}$ & $\begin{array}{l}E_{c}{ }^{4} \\
88 \%\end{array}$ \\
\hline & & $\begin{array}{l}\text { 2. Min. Rated Capacity }{ }^{2} \\
\text { Steady-State }\end{array}$ & $\begin{array}{l}\mathrm{E}_{\mathrm{c}}^{4} \\
88 \%\end{array}$ \\
\hline \multirow{2}{*}{$\begin{array}{c}\text { U.L. 726-75 } \\
\text { H.I. Htg. Boiler Std. } 86 \\
\text { ASME PTC4.1-64 }\end{array}$} & \multirow[t]{2}{*}{$\begin{array}{c}\text { Oil-Fired } \\
\geq 300,000 \mathrm{Btu} / \mathrm{h}\end{array}$} & $\begin{array}{l}\text { Max. Rated Capacity }{ }^{2} \\
\text { Steady-State }\end{array}$ & $\mathrm{E}_{c^{4}}{ }^{4}$ \\
\hline & & $\begin{array}{l}\text { 2. Min. Rated Capacity } \\
\text { Steady-State }\end{array}$ & $\begin{array}{l}E_{c}^{4} \\
91 \%\end{array}$ \\
\hline \multirow{2}{*}{$\begin{array}{l}\text { H.I. Htg. Boiler Std. } 86 \\
\text { ASME PTC4.1-64 }\end{array}$} & \multirow{2}{*}{$\begin{array}{l}\text { Oil-Fired (Residual) } \\
\geq 300,000 \mathrm{Btu} / \mathrm{h}\end{array}$} & $\begin{array}{l}\text { 1. Max. Rated Capacity }{ }^{2} \\
\text { Steady-State }\end{array}$ & $\begin{array}{l}E_{c}^{4} \\
91 \%\end{array}$ \\
\hline & & $\begin{array}{l}\text { 2. Min. Rated Capacity }{ }^{2} \\
\text { Steady-State }\end{array}$ & $\begin{array}{l}E_{c}^{4} \\
91 \%\end{array}$ \\
\hline
\end{tabular}

1. To be consistent with National Appliance Energy Conservation Act of 1987 (P.L. 100-12).

2. Provided and allowed by the controls.

3. Except for gas-fired steam boilers for which minimum AFUE is $83 \%$.

4. $E_{C}=$ combustion efficiency, $100 \%$ - flue losses. See reference document for detailed information. 


\section{B. Outdoor Air Ventilation Requirements}

A building must be exhausted of excess carbon dioxide, water vapor, odors, and air pollutants that accumulate in a building. Of course, an equal amount of fresh air must be introduced to replace the exhausted air. Special concern should be placed on the interior building pressures of adjacent spaces such as a garage and office space. In this example, it is important that the office be pressurized more than the garage to prevent vehicle exhaust fumes or other contaminants from spreading into the office space. At all times buildings should be designed to be slightly above atmospheric pressure in order to prevent infiltration of untreated air through normal building penetrations such as building joints.

Table 5

Outdoor Air Requirements for Ventilation DOT Facilities

- (Excerpts from the Washington State Ventilation and Indoor Air Quality Code)

\begin{tabular}{|c|c|c|}
\hline Application & Estimated Maximum & 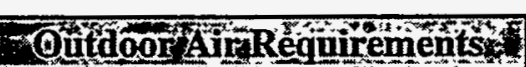 \\
\hline $7 \sqrt{t+6}$ & sececipancy & crmplenson (or as o them \\
\hline$x^{2}$ & Personsicootifer & 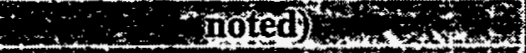 \\
\hline 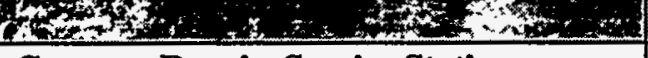 & $x+3=100 \mathrm{~m}^{2}+2=2$ & 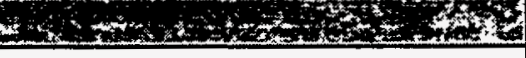 \\
\hline \multicolumn{3}{|l|}{ Garages, Repair, Service Stations } \\
\hline Enclosed parking garage & & $1.50 \mathrm{cfm} / \mathrm{ft} . \mathrm{sq}$. \\
\hline Auto repair rooms & & $1.50 \mathrm{cfm} / \mathrm{ft} . \mathrm{sq}$. \\
\hline \multicolumn{3}{|l|}{ Offices } \\
\hline Office space & 7 & 20 \\
\hline Reception area & 60 & 15 \\
\hline $\begin{array}{l}\text { Telecommunication centers and data entry } \\
\text { areas }\end{array}$ & 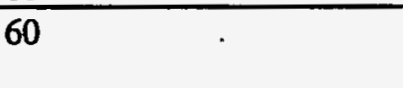 & 20 \\
\hline Conference rooms & 50 & 20 \\
\hline \multicolumn{3}{|l|}{ Public Spaces } \\
\hline Corridors and utilities & & $0.005 \mathrm{cfm} / \mathrm{fts}$ \\
\hline Public restroom & & $50 \mathrm{cfm} / \mathrm{wc}$ or urinal \\
\hline Lockers and dressing rooms & & $.05 \mathrm{cfm} / \mathrm{ft} . \mathrm{sq}$. \\
\hline Smoking lounge & 70 & 60 \\
\hline Elevators & & $1.0 \mathrm{cfm} / \mathrm{ft} . \mathrm{sq}$. \\
\hline \multicolumn{3}{|l|}{ Transportation } \\
\hline Waiting rooms & 100 & 15 \\
\hline Platforms & 100 & 15 \\
\hline Vehicles & 150 & 15 \\
\hline \multicolumn{3}{|l|}{ Education } \\
\hline Classroom & 50 & 15 \\
\hline Laboratories & 30 & 20 \\
\hline
\end{tabular}




\section{Heat Recovery}

It is recommended that DOT facilities follow section 1436 of the WSEC in regard to heat recovery.

1436 Heat Recovery: Fan systems which have both a capacity of 5,000 cfm or greater and which have a minimum outside air supply of $70 \%$ or greater of the total air circulation shall have a heat recovery system with at least $50 \%$ recovery effectiveness. Fifty percent heat recovery effectiveness shall mean an increase in the outside air supply temperature at design heating conditions of one half the difference between the outdoor design air temperature and $65^{\circ} \mathrm{F}$. Provision shall be made to bypass or control the heat recovery system to permit air economizer operation as required by Section 1433 . Heat recovery energy may be provided from any site-recovered or site-solar source.

\section{Exceptions:}

1. Laboratory systems equipped with both variable air volume supply and variable air volume or two-speed exhaust fume hoods.

2. Systems serving spaces heated to less than $60^{\circ} \mathrm{F}$.

3. Systems which can be shown to use as much energy with the addition of heat recovery equipment as without it.

4. Systems exhausting toxic, flammable, paint exhaust or corrosive fumes making the installation of heat recovery equipment impractical.

5. Type I commercial kitchen hoods.

\section{Special HVAC Considerations for Offices}

Packaged HVAC equipment should be considered for small offices. Air-to-air heat pumps can be a good choice where electricity costs are low and gas costs relatively high. When both gas and electricity are available, dual-fuel heat pumps can be a good choice. In some cases a designer should consider a ground-coupled heat pump system if gas is not available and electricity costs are high.

Office designs can conserve heat by incorporating vestibule entries. Vestibule heaters do improve comfort to adjacent interior spaces but use additional energy.

\section{E. Special HVAC Considerations for Shops}

Use of infrared radiant and hydronic slab heating should be considered in shops where feasible. These systems are more economical in applications where high infiltration loads are present; the thermostat can generally be set 5-6 degrees lower than a forced air system and provide comparable comfort levels.

Designers should use ventilation or evaporative cooling (swamp coolers) for general shop areas. Mechanical cooling of shop areas should be limited to the central desert areas of the state or to functions for which temperature and/or humidity control are vital. 


\section{F. Fuel Selection}

When natural gas is not available, air-to-air heat pumps with electric backup are one possible choice, particularly for office areas. Propane fuel is another option that should be investigated, particularly where electric costs are high and/or if a shop or storage space is to be heated. Propane can serve either a hydronic boiler or radiant heat application.

To determine the best current selection for fuel, you may wish to use Table 6 , "Comparing Fuel Costs." This table reduces fuel costs to a common denominator, dollars per million Btu's. To use this table, just follow across from the fuel type to the price for the respective fuel in question. Then read relative fuel cost at the bottom of the table. The heat pump selection assumes a coefficient of performance of 2 . This is about right for air-to-air systems, but may be closer to 4 for earth-coupled heat pumps. 


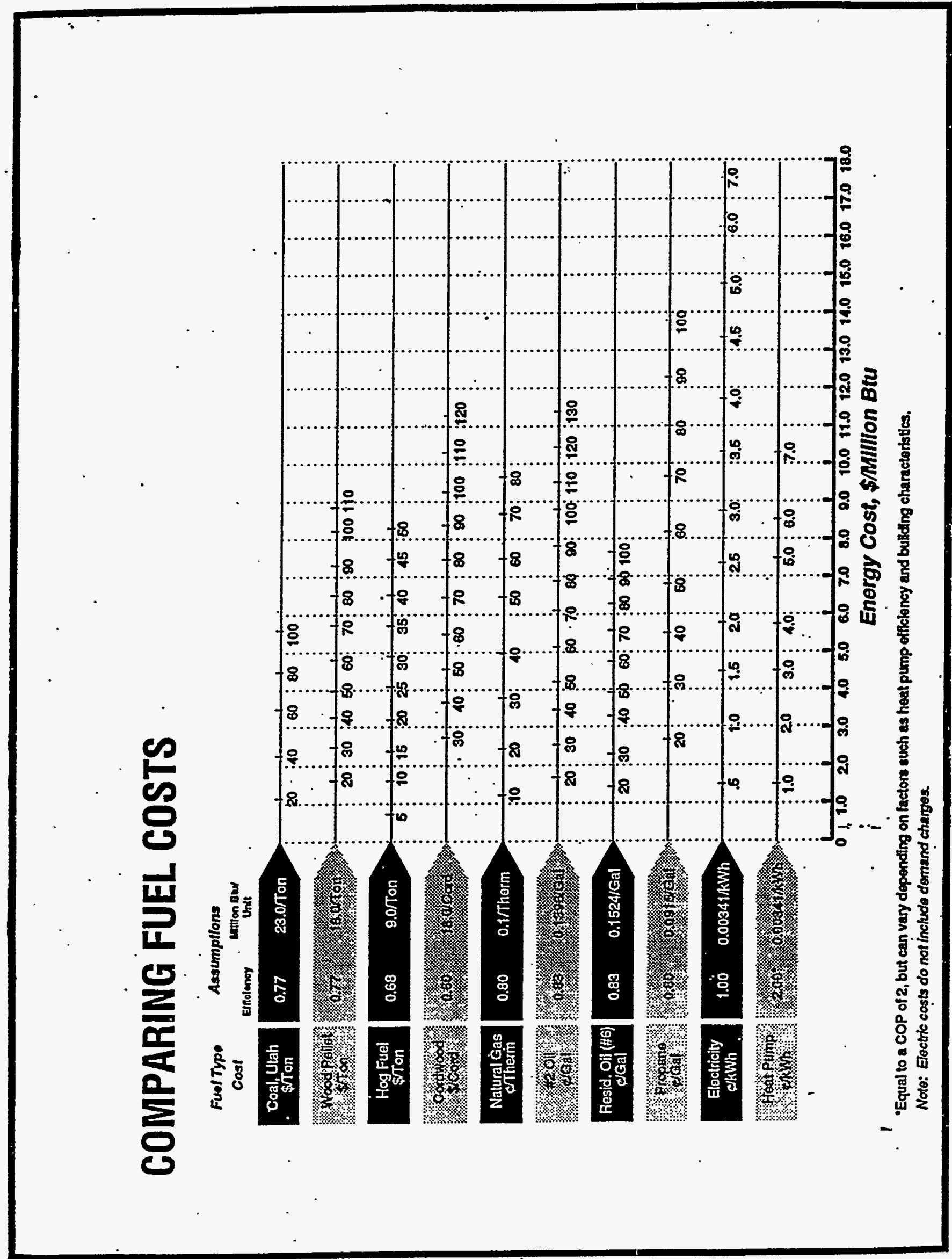




\section{G. Other Considerations in Selecting HVAC Systems .}

\section{Equipment Sizing}

Sizing HVAC equipment is both an art and a science. The Washington State Energy Code no longer requires that sizing calculations be submitted to planning departments. It is advisable to have designers submit equipment sizing calculations with design development submittals. The method should be similar to that described in ASHRAE Fundamentals Chapter 26. Designers rely on experience or "rules of thumb" approaches to the load.

Many improperly-sized systems are installed, generally on "the side of caution." This results in excessive cycling, increased operating costs, and higher first cost.

\section{Staging Equipment}

Boilers and chillers can be installed in pairs and designed to operate in stages. Generally, the more fully loaded a piece of equipment is, the more efficient it operates. For example, two boilers each capable of $75 \%$ of the peak load can be installed and controlled so that the lead boiler will operate at $100 \%$ capacity before the second boiler comes on-line.

\section{Air Conditioning Equipment}

A choice often needs to be made between installing a central cooling plant or individual packaged equipment. A chiller generally affords better reliability and higher efficiency than a collection of smaller selfcontained units. The best way to decide is with a full Life Cycle Cost Analysis. The estimated cooling load and usage play a large role in the economics of a central cooling plant.

Packaged rooftop HVAC equipment over 4-1/2 tons of cooling $(54,000$ BTUH) will have an airside economizer, required by WSEC 1423 and 1433. Economizers introduce outdoor air for "free" cooling. The economizer controls are to be of the integrated type, meaning the unit must operate to maintain the lowest possible return temperature so long as icing conditions do not occur at the evaporator coil. Adequate relief opening must be present in the system to allow this excess outdoor air to escape. Buildings that are heated only, can benefit from a similar arrangement, which is simply referred to as ventilation cooling.

It is also advisable to include both low and high pressure switches on refrigeration equipment to avoid serious equipment failures when maintenance is needed. 


\section{Infrared Heaters}

Infrared radiant heaters directly heat objects or people as opposed to heating the atmosphere. They do this by emitting infrared radiation. For applications such as shop areas where infiltration loads are high, WSEO recommends designers consider this technology.

Radiant heaters typically come in two types: tube (low intensity) and ceramic (high intensity). These heaters can be fueled by either natural gas or propane. These heaters are usually mounted from the ceiling and work well in large open spaces. In most cases a ventilation system of some type will be necessary to augment this system. Radiant heaters create an average radiant temperature equivalent to a forced air system set 5 degrees warmer; this means that, at 60 degrees, workers feel similar comfort levels to $65 \mathrm{~F}$ with a conventional system.

\section{Other Radiant Heaters}

Hydronic slab heating is another form of radiant heating that would work equally well in shop areas. The materials now available have made these systems excellent performers in both commercial and residential applications. Improved comfort and energy efficiency are the real selling points of these systems. The main fuel source to heat the water can be propane, natural gas, or heat pumps. An electric boiler would probably prove to be too expensive in most parts of the state.

\section{Hydronic Heat Pumps}

Generally WSEO does not recommend this type of system except when ground coupled. The ground-coupled system should not be installed without careful analysis of alternatives. Ground-coupled systems are generally among the most energy efficient and are often recommended in areas where gas is unavailable for the foreseeable future; however, a life cycle cost analysis should carefully weigh their high first cost against their lower operating cost.

\section{H. Suggested HVAC Configurations}

Table 7 provides suggested HVAC system types and controls for offices, shops and storage buildings. These system recommendations are based on collective experiences with similar buildings analyzed at WSEO. 


\begin{tabular}{|c|c|c|c|c|}
\hline \multicolumn{5}{|c|}{ Offices with some shops and storage ${ }^{(1)}$} \\
\hline $\begin{array}{c}\text { Option } \\
A\end{array}$ & $\begin{array}{c}\text { Individual fan } \\
\text { coils and unit } \\
\text { heaters } \\
\end{array}$ & 85\%-efficient gas boiler & $\begin{array}{c}\text { Air or evaporative } \\
\text { cooled chiller w/ } \\
\text { economizer }\end{array}$ & $\begin{array}{l}\text { Chiller lock out, reset hot water } \\
\text { temps, } \\
\text { fully integrated economizer }{ }^{(3)} \text {. }\end{array}$ \\
\hline $\begin{array}{c}\text { Option } \\
B\end{array}$ & $\begin{array}{l}\text { Rooftop } \\
\text { packaged } \\
\text { multizone }\end{array}$ & $\begin{array}{l}85 \% \text {-efficient gas boiler } \\
\text { or furnace }\end{array}$ & $\begin{array}{l}\text { Direct expansion } \\
\text { w/ economizer or } \\
\text { chiller }\end{array}$ & $\begin{array}{l}\text { Fully integrated economizer, } 7 \text { - } \\
\text { day programmable thermostat }\end{array}$ \\
\hline \multicolumn{5}{|c|}{ Office only ${ }^{(2)}$} \\
\hline $\begin{array}{c}\text { Option } \\
A\end{array}$ & $\begin{array}{l}\text { Rooftop single } \\
\text { zone }\end{array}$ & $\begin{array}{l}\text { Rooftop } \\
\text { gas furnace with direct } \\
\text { expansion cooling or } \\
\text { heat pump }\end{array}$ & $\begin{array}{l}\text { Direct expansion } \\
\text { w/ economizer }\end{array}$ & $\begin{array}{l}\text { Fully integrated economizer. } \\
\mathrm{VVVT}^{(4)} \text { controls optional }\end{array}$ \\
\hline$\overline{\text { Option }}$ & $\begin{array}{l}\text { Hydronic } \\
\text { Heatpumps }\end{array}$ & $\begin{array}{l}\text { Ground coupled (open } \\
\text { or closed loop) }\end{array}$ & $\begin{array}{c}\text { Ground coupled } \\
\text { (open or closed loop) }\end{array}$ & $\begin{array}{l}\text { Fully integrated economizer. } 7- \\
\text { day programmable thermostat }\end{array}$ \\
\hline \multicolumn{5}{|c|}{ Shops } \\
\hline $\begin{array}{c}\text { Option } \\
A\end{array}$ & $\begin{array}{l}\text { Overhead } \\
\text { radiant heaters, } \\
\text { auxiliary } \\
\text { ventilation }\end{array}$ & $\begin{array}{l}\text { Suspended fully vented } \\
\text { gas radiant heaters. } \\
\text { Consider } 85 \% \\
\text { efficiency or better }\end{array}$ & $\begin{array}{l}\text { Ventilation only, } \\
\text { evaporative cooling } \\
\text { Zone } 2\end{array}$ & $\begin{array}{l}\text { Interlock heat with door controls, } \\
\text { exhaust heat recovery, positive } \\
\text { closing HVAC vents }\end{array}$ \\
\hline $\begin{array}{c}\text { Option } \\
B\end{array}$ & $\begin{array}{l}\text { In-slab radiant } \\
\text { heat, auxiliary } \\
\text { ventilation }\end{array}$ & $\begin{array}{l}85 \% \text { - efficient gas } \\
\text { boiler }\end{array}$ & $\begin{array}{c}\text { Ventilation only, } \\
\text { evaporative cooling } \\
\text { Zone } 2\end{array}$ & $\begin{array}{c}\text { Interlock heat with door controls, } \\
\text { exhaust heat recovery, positive } \\
\text { closing HVAC vents }\end{array}$ \\
\hline \multicolumn{5}{|c|}{ Storage Buildings (heated-only) } \\
\hline & $\begin{array}{l}\text { Furnaces, } \\
\text { boilers or unit } \\
\text { heaters }\end{array}$ & $\begin{array}{l}85 \% \text { - efficient furnace, } \\
\text { boiler or unit heaters }\end{array}$ & Ventilation only & $\begin{array}{l}\text { 7-day programmable thermostat, } \\
\text { positive closing HVAC vents }\end{array}$ \\
\hline
\end{tabular}

(1) These systems are desirable for larger buildings with gas availability.

(2) These systems are desirable for small- to medium-sized, single-story facilities.

(3) Air economizers shall be capable of providing partial cooling even when additional mechanical cooling is required to meet the remainder of the cooling load. See Section 1413.3 of the WSEC for further description.

(4) VVVT is "variable volume/variable temperature." 
1 


\section{Lighting}

\section{A. Lighting Densities and Light Levels}

Following are recommended lighting densities based on chapter 15 of the WSEC. The light levels are IES, Iluminating Engineering Society recommendations.

\begin{tabular}{|c|c|c|}
\hline Buifoing Type & $\begin{array}{l}\text { Aghting Density Wisgets } \\
\text { (from WSECGhapter 15) }\end{array}$ & 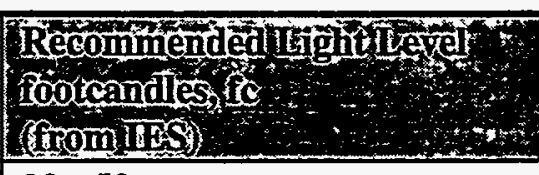 \\
\hline Offices & 1.2 & $30-50$ \\
\hline Laboratories & 2.0 & $75-100$ \\
\hline Garages/Shops-General & 1.5 & $30-50$ \\
\hline $\begin{array}{l}\text { Painting, Welding, Carpentry, } \\
\text { Machine Shops }\end{array}$ & 2.3 & $30-75$ \\
\hline Warehouses, Storage Areas & 0.5 & $7.5-10$ \\
\hline
\end{tabular}

\section{B. Specific Lighting Recommendations for Offices}

Office lighting strategies include using electronic ballasts and T-8 fluorescent lamps.

Lighting level controls are often used to decrease glare on computer screens by lowering light levels. Other ways to reduce glare would be selecting parabolic reflectors specifically designed for computer applications.

Energy code now requires automatic shutoff controls and separate light switches in offices within 10 feet of window walls or skylights.

\section{Specific Recommendations for Shop Lighting}

It is recommended that shop lighting be T-8 fluorescent or metal halide fixtures. Many existing shops currently utilize 8-foot, HO lamps. These systems can consume $30 \%$ more electricity than T-8 or metal halide technology. Another advantage of metal halide fixtures is they provide a point source of light which can enhance depth or texture perception.

Surfaces of the lamp and reflector often become soiled and unreflective, affecting light output. An enclosed fixture is a better choice because it decreases dirt accumulation.

When locating fixtures, special consideration needs to be given to the presence of any obstructions such as hoists, ductwork, radiant heaters, conduits and piping systems. 
Another lighting concern besides energy efficiency is employee safety. Florescent lamps operated from magnetic ballasts may flicker or produce what is called stroboscopic effect. This can be a safety hazard in shops that utilize rotating machinery. Machinery that is moving at a uniform speed may appear to move jerkily or may even appear to be standing still or rotating in a reverse direction. Electronic ballasts that utilize very high frequencies eliminate this effect. Special attention should be given to specify quality ballasts. Light levels should be carefully analyzed.

\section{Specific Recommendation for Exterior Lighting}

The main objective of exterior lighting is to provide security and safety. This objective can be met while also reducing exterior lighting costs.

Any new or remodeled facility should adhere to Section 1532 of the WSEC. The lighting allowance for parking areas should be $0.20 \mathrm{~W} / \mathrm{sq}$.ft. Allowances for building exteriors should be calculated by either multiplying the building facade area by $0.25 \mathrm{~W} / \mathrm{sq}$.ft. or multiplying the building perimeter in feet by 7.5 watts per lineal foot.

Existing facilities should consider lighting re-design if the following conditions exist:
$\checkmark$. Current light levels are not uniform.
$\checkmark \quad$ Light levels are too high or too low.
$\checkmark \quad$ Lighting poles are rotted or wiring is failing.
$\checkmark \quad$ Current lights are standard incandescent, tungsten halogen or mercury vapor.

All replacement lights should be high pressure sodium type. These lights perform very well because of their higher efficacy (lumens per watt). It is important to carefully consider lighting placement rather than simply replacing old lights on a one-for-one basis. Light mounting height is equally important. Most vendors are very good at making proper recommendations.

For large installations, it is desirable to compare several options on a life-cyclecost basis. Variable factors include fixture type, wattage, pole height, maintenance cost, operating expenses and installation costs.

All exterior lighting should have a photocell or timeclock control. This will prevent systems from being left on during the day. Another strategy is to rewire for flexible switching. This provides separate circuits for the security lights, building perimeter lights and general parking lights 


\section{Building Commissioning}

Many utilities and governments are realizing the benefits of commissioning.

Commissioning of new and existing buildings is a method to ensure that all building systems meet the design intent and their owners' operational requirements. The concept of commissioning evolved during the 1980 s to respond to the then-growing problem of poor indoor air quality (IAQ). Since then the definition has broadened and can even be described as "quality assurance" practices.

The Bonneville Power Administration defines commissioning as,

A process for achieving, verifying and documenting that the performance of a building and its various systems meet the design intent and the owner and occupants' operational needs. The process ideally extends through all phases of a project from concept to occupancy and operation.

WSEO would like to expand the definition above to include the preparation of building operation personnel. In September of 1993 the State of Washington General Services Administration added commissioning guidelines to their buildings-program. WSEO suggests DOT consider commissioning in their standard procedures for building construction. 



\section{General Issues}

\section{A. Resource Management}

The key to efficient operation of any building is proper resource management. Resource management is as important as initial facility design. The first step to good resource management is designating responsibility. The resource manager monitors expenditures including water, sewer, garbage pickup, and energy. State agencies can effectively monitor usage by using available energy management software, which can readily make comparisons between one year and the next. Resource management is beyond the scope of these guidelines; however, WSEO can be contacted for more information.

\section{B. Co-locating}

Combining offices of several state or local government agencies on one site is becoming more common. Co-locating presents an opportunity for public agencies to share resources. When entering into a co-location project, agencies should not approach it as a governmental "strip mall." Rather, to maximize the benefit, projects should be integrated to the degree practical in the design phase. Savings can be achieved by having buildings share mechanical and electrical services and systems. Not only can this lower the per square foot cost of the new construction, but the annual cost of services can be lowered as well.

\section{Equipment Replacement-}

Replacing equipment is a savings opportunity that is often overlooked. New equipment can often achieve a $30 \%$ to $40 \%$ savings over what is in place, as well as make significant improvements to human comfort. Also, when replacing equipment, attention should be given to proper equipment sizing for maximum benefits. 


\section{Summary}

In addition to providing the above energy efficiency guidelines, the Washington State Energy Office is a resource that can be helpful to DOT on a variety of energy-related issues including building operator training, energy policy, energy programs, and information services.

For information on the range of services offered by WSEO, please contact your WSEO program representative. 


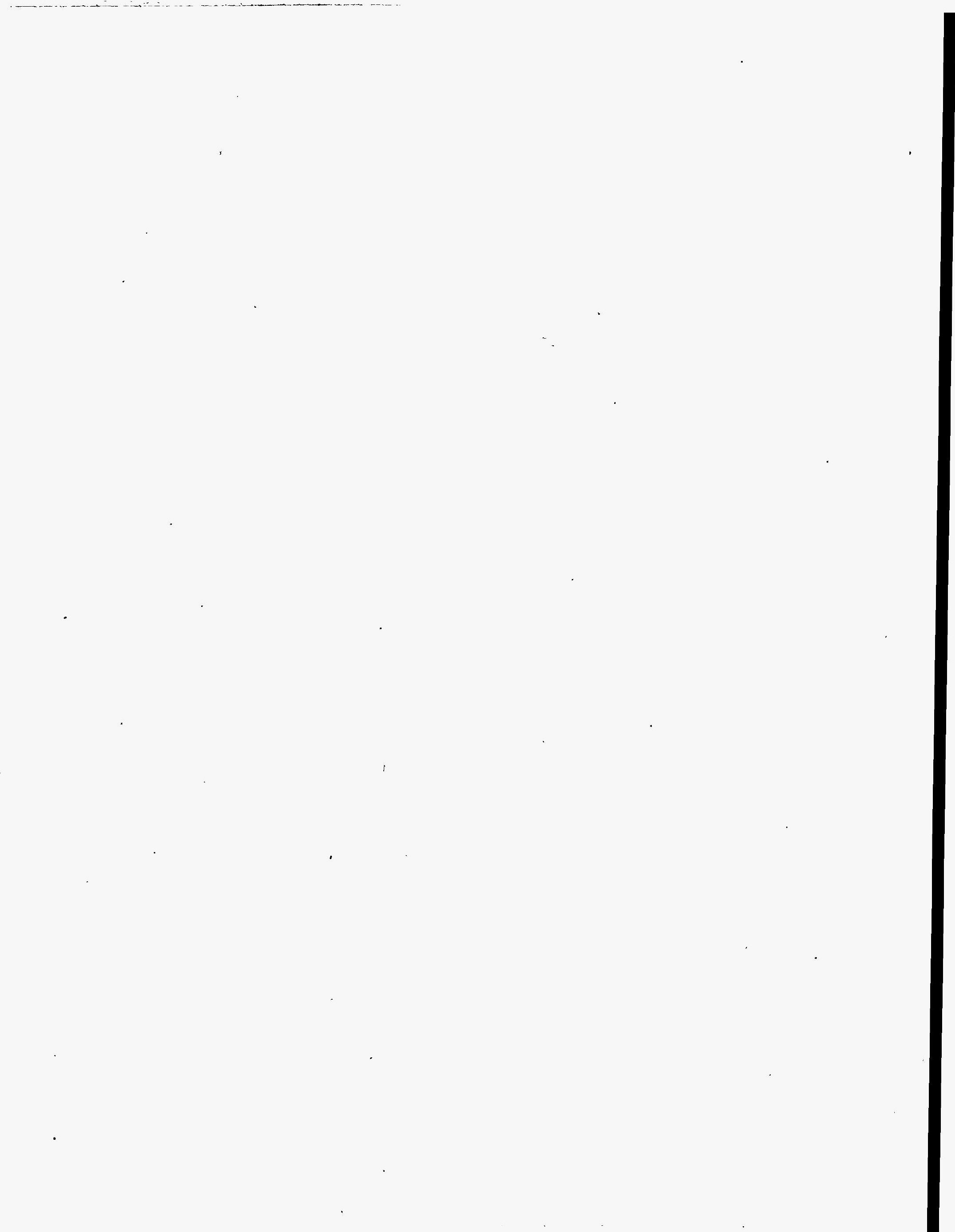

